\title{
Tendencias de la segregación residencial en León, Guanajuato (1990-2010)
}

Recibido: 30/06/2015 · Aceptado: 24/07/2015

Javier Tun*

Brigitte Lamy

Universidad de Guanajuato, Campus León

\section{Resumen}

De acuerdo con investigaciones recientes las tendencias de la segregación residencial en las ciudades latinoamericanas van en dos sentidos principalmente: en un cambio hacia una menor escala de observación y hacia la intensificación de los efectos negativos, sobre todo en la población de menores recursos. Este trabajo examina las tendencias de la segregación residencial en León, Guanajuato, durante el periodo 1990-2010, como un ejemplo de ciudad media mexicana, para verificar si estas se inscriben dentro del modelo latinoamericano reciente y advertir otros aspectos de estos procesos de configuración del espacio urbano. Con tal propósito se realizan análisis factoriales por componentes principales para los años 1990, 2000 y 2010 con información censal en el nivel de área geoestadística básica (АСЕB), lo que permite conocer la configuración de la ciudad a partir de la definición de cinco estratos socioespaciales. Los resultados muestran que las dos tendencias se cumplen para León, además de que el proceso de dispersión urbana sigue siendo característico de esta ciudad. Ello se comprueba, en primer lugar, porque las grandes zonas tradicionalmente identificadas como de estratos altos, medios o bajos están incorporando poblaciones de diferentes estratos; en segundo lugar, porque las distancias entre los valores medios de los estratos altos y bajos van en aumento, y, finalmente, por un crecimiento más acelerado de la mancha urbana en relación con la población y una disminución del promedio de habitantes por área geoestadística básica.

Palabras clave: Análisis factorial, ciudad de León, configuración del espacio, estratificación socioespacial, segregación residencial.

*Correo electrónico: javierlmj@gmail.com 


\title{
Trends of residential segregation in León, Guanajuato (1990-2010)
}

\author{
Javier Tun* \\ Brigitte Lamy \\ Universidad de Guanajuato, Campus León
}

\begin{abstract}
Recent research suggests that the trends of residential segregation in Latin American cities follow two main tendencies, in a shift towards smaller scale of observation, and to the intensification of the negative effects especially on low-income population. The aim of this paper is to analyze the trends of residential segregation in the city of León, Guanajuato during the 1990-2010 period, as an example of a Mexican middle size city, to verify if these trends fall within the recent Latin American model and record other aspects of these processes of urban space configuration. For this purpose, factorial analysis by principal components for the years 1990, 2000 and 2010 were carried out using census data at the level of Basic Geo-statistical Area (AGEB), which allows knowing the configuration of the city from the definition of five socio-spatial strata. Results show that both trends hold true for León, in addition that the process of urban dispersion remains characteristic of this city. This is confirmed, first because the large areas traditionally identified as high, medium or low strata are incorporating populations of different strata; secondly because the distances between the mean values of the upper and lower layers are increasing; and finally by the faster growth of the urban area in relation to the population and a reduction in the average population by Basic Geostatistical Area.
\end{abstract}

KEY words: Factorial analysis, city of León, space configuration, socio-spatial stratification, residential segregation.

*E-mail: javierlmj@gmail.com

TeORIA y PRAXIS · ISSN 18701582 • nÚM. 20 · JULIO-DICIEMBRE 2016 · 37-67 pp. 


\section{Introducción}

El proceso de urbanización y la creciente importancia de las ciudades han sido dos de las características más relevantes de la sociedad moderna. Hace algunos años se hablaba de que al inicio del presente siglo más de la mitad de la población habitaría en ciudades, principalmente por la alta urbanización de los países en desarrollo. Ahora se prevé que para 2030 todas las regiones, incluso las menos desarrolladas en Asia y África, tendrán más población viviendo en ciudades que en zonas rurales (UN-Habitat, 2008).

Diferentes autores coinciden en que los grandes cambios en la estructura económica, que han transformado el mundo en las últimas décadas, también han modificado el esquema espacial de las ciudades; sin embargo, cabe preguntarse en qué medida, de qué manera y con qué tiempos se dan estas transformaciones, pero también cómo las peculiaridades de la organización del espacio urbano pueden influir en la sociedad y en la vida de las familias (Marcuse y Van Kempen, 2000; Goldsmith, 2000, y Maurin, 2004, citados en Schteingart, 2010).

La segregación residencial es una manifestación de esta división social del espacio que puede ayudar a comprender algunos de estos procesos y al mismo tiempo las afectaciones a la sociedad y el medio ambiente. A partir del análisis de la segregación residencial es posible advertir algunos procesos de la configuración del espacio al interior de las ciudades a diferente escala de observación, lo cual se pretende abordar en este artículo.

El trabajo presenta un análisis factorial por componentes principales en la ciudad de León, Guanajuato, para 1990, 2000 y 2010, con lo que se busca identificar las tendencias primordiales de la segregación residencial y los cambios en la configuración del espacio urbano. En primer lugar se expondrán los antecedentes en el estudio de la segregación residencial, para después mostrar la metodología utilizada, los resultados para los tres momentos mencionados y una discusión acerca de los hallazgos. En los comentarios finales se habla de cómo estas tendencias encontradas en León se enmarcan en aquellas más importantes recientemente observadas en las ciudades latinoamericanas. 


\section{Antecedentes}

La noción de segregación dentro de la sociología urbana remite a los fenómenos de concentración de los grupos sociales homogéneos en el espacio urbano y su distanciamiento de otros grupos distintos (McKenzie, 1967); sin embargo, es mucho más complejo que una cuestión de localización de grupos sociales o espacialización de la estructura social, ya que intervienen relaciones entre la estructura social, cuestiones determinantes económicas, políticas y las preferencias de los individuos y familias en la configuración de esta división socioespacial en las ciudades (Castells, 1976; Rubalcava y Schteingart, 2012). La segregación residencial se ha vinculado con fenómenos como la pobreza, la exclusión social y la fragmentación urbana, por mencionar algunos, y ha sido un tema de estudio también dentro de la economía y la geografía, entre otras disciplinas. El trabajo se enfoca en revisar los antecedentes en la sociología urbana, área donde este se inscribe.

Los primeros antecedentes se encuentran a inicios del siglo xx en la escuela de Chicago, ya que fue de las primeras en abordar las interrelaciones del hombre y su entorno a través de la ecología urbana (Duhau, 2003), teniendo como objeto de estudio a la ciudad y las relaciones sociales que en ella suceden, utilizando métodos empíricos y técnicas estadísticas especializadas.

Más en específico, la segregación residencial -sus orígenes, magnitud, dinámica y consecuencias- ha sido largamente abordada por la investigación social debido a su pertinencia para evaluar los niveles de integración y equidad que alcanzan las sociedades (Groisman, 2010). Se han realizado esfuerzos relevantes para su medición, además de estudiar los diferentes elementos que intervienen en la segregación; los trabajos también se han enfocado en la combinación de indicadores cuantitativos y técnicas cartográficas y/o en sus fundamentos microeconómicos.

En América Latina estos estudios se intensificaron sobre todo con la disponibilidad de fuentes de datos a escalas pequeñas, básicamente provenientes de los censos de población. Por lo general se ha empleado el análisis factorial u otras técnicas estadísticas similares para establecer dimensiones y, en sentido estadístico, examinar las diferencias en la distribución de un conjunto de variables mediante las cuales son comparadas entre sí las unidades territoriales sometidas a revisión (Duhau, 2003). Algunos trabajos al respecto son los de 
Rubalcava y Schteingart (2000), Preteceille y De Queiroz Ribeiro (1999), González Arellano y Villeneuve (2002), Aliaga y Álvarez (2010) y Aguilar y Mateos (2011), entre otros.

Desde el inicio ha habido varias persectivas desde las cuales estudiar este fenómeno, no siempre usando el término de segregación, pero sí haciendo referencia a los procesos de localización socioespacial de la población y de las relaciones entre actores sociales que configuran estas formas de producción y apropiación del espacio urbano habitable.

Así, se han utilizado distintos enfoques de aproximación a la segregación. El de marginalidad urbana la interpretó como una condición de exclusión: de las ventajas del sector moderno en la vertiente funcionalista (Germani, 1973), y del sector económico-social-político dominante y formal en la versión dependentista (Quijano, 1977). En el enfoque neoclásico del análisis urbano, la segregación es producto de condiciones sociales exógenas a, y operando sobre, los mecanismos de mercado que determinan la localización residencial (Alonso, 1988 cit. en Alegría, 1994). En el enfoque neomarxista predominante, la segregación entre grupos sociales es resultado del ejercicio del poder de la clase dominante sobre los mecanismos del mercado inmobiliario (Harvey, 2005) para obtener ganancias y asegurar la reproducción del sistema. En esta perspectiva se vislumbra la separación conceptual de segregación y localización, sin embargo, tal separación no es desarrollada (Alegría, 1994).

Por otro lado, se ha llegado a un acuerdo en que la segregación residencial se manifiesta de tres maneras (Arriagada y Rodríguez Vignoli, 2003): a) la proximidad física entre los espacios residenciales de los diferentes grupos sociales; b) la homogeneidad social de las distintas subdivisiones territoriales en que se puede estructurar una ciudad, y c) la concentración de grupos sociales en zonas específicas de una ciudad.

No obstante, hay otros aspectos del fenómeno que poco se han explorado. Por ejemplo, existen escasos análisis comparativos entre países, o en torno a procesos de evolución de la segregación en periodos relativamente largos. Asimismo, más allá de generar algunas hipótesis sobre efectos de la segregación, poco se le ha podido correlacionar con otros fenómenos como la violencia urbana, el mercado laboral, la educación o el capital social. 
De acuerdo con Schteingart (2001), la problemática de la estratificación socioespacial en los países y en las ciudades estuvo presente en una cantidad no insignificante de estudios en América Latina, sobre todo en los años sesenta y setenta. Después hubo una especie de olvido acerca del tema en términos globales, para enfocarse a casos concretos sobre ciertos fenómenos. De ello dan cuenta los balances de Valladares y Prates (1995) y Schteingart (1995), por ejemplo.

A partir de la década de 1990, los investigadores latinoamericanos han vuelto a poner su atención en la temática. Schteingart (2001), Rodríguez Vignoli (2001), Sabatini (2003), Aliaga y Álvarez (2010) y Groisman (2010), entre otros, coinciden en un renovado interés por el estudio de la segregación residencial o temas conexos. En América Latina, las preocupaciones han estado más hacia la concentración espacial de la pobreza y sus efectos (Rubalcava y Schteingart, 2001; González Arellano y Villeneuve, 2002; Roberts y Wilson, 2009; Rodríguez Vignoli y Arriagada, 2004; Sabatini y Brain, 2008, por citar algunos).

Esta reciente importancia del tema es atribuible a diversos factores. Por ejemplo, Schteingart (2001) señala que a raíz de la disponibilidad de datos estadísticos desagregados en unidades de análisis pequeñas, los estudios de este tipo han proliferado; Aliaga y Álvarez (2010) mencionan que Estados Unidos ha tenido una gran influencia al difundir sus investigaciones hacia América Latina a partir de los años noventa, principalmente sobre cuestiones teóricas y empíricas.

Siguiendo con Schteingart (2001), en México estos trabajos se pueden agrupar dentro de cinco categorías analíticas, con base en el énfasis temático que presenta cada uno de ellos: 1) la segregación relacionada con la expansión de las ciudades y el crecimiento de la población; 2) la segregación vinculada con los servicios urbanos y la vialidad; 3) los aspectos históricos de la segregación residencial; 4) estudios específicos de algunas partes de la ciudad: el centro, los barrios, la periferia irregular, y 5) las nuevas formas de segregación urbana, como los barrios cerrados. Este trabajo se enmarca dentro de la primera categoría, haciendo énfasis en los cambios que la configuración del espacio urbano está teniendo en las últimas décadas. 


\section{Metodología}

La dimensión objetiva de la segregación es la corriente con mayor tradición dentro del análisis de este fenómeno en los estudios urbanos; en términos generales se pueden identificar dos aspectos principales de acuerdo con Sabatini (2003): la tendencia de los grupos sociales a concentrarse en algunas zonas de la ciudad y la conformación de áreas o barrios socialmente homogéneos.

A fin de realizar el análisis de la segregación residencial para las últimas décadas se utilizan como fuente de información los censos de población y vivienda en el nivel de Área Geoestadística Básica (AGEB). Según la disponibilidad de información, se determinó iniciar a partir de la década de 1990, ya que el censo de ese año fue el primero en contemplar el nivel de AGEB, empleando así los censos de 1990, 2000 y 2010. La unidad de observación es la ciudad de León, que se ha definido como el conjunto de localidades urbanas (de más de 2500 habitantes) del municipio de León, Guanajuato.

Con base en la revisión de técnicas de análisis en la bibliografía consultada, destacan los trabajos de Rubalcava y Schteingart (2012), entre otros, que usan la técnica de análisis factorial por componentes principales, por la cual se ha optado, ya que puede tomar diversas variables y sintetizarlas en pocos componentes. Estos análisis se realizan para los tres momentos ya mencionados, utilizando variables físicomateriales de las viviendas y socioeconómicas de la población.

En la representación espacial de la información se emplea el método cartográfico, que consiste en la elaboración de mapas para comunicar, interpretar y comprender diferentes hechos y fenómenos, los elementos o variables de estos y sus relaciones en el espacio (Gómez, 2004). El método de representación cartográfica utilizado es de áreas. Los mapas se han podido elaborar para los años 2000 y 2010, ya que para 1990 no fue posible conseguir la información estadística georreferenciada.

Para este y otros tratamientos cuantitativos y de información se ha seleccionado el programa estadístico SPSS, y para la representación espacial de los datos se desarrolló un sistema de información geográfica usando el programa QGIS.

\section{Resultados: la segregación residencial en la ciudad de León, 1990-2010}

El modelo de componentes principales integra siete variables que representan las dimensiones teóricas de la segregación residencial y una valoración estadística 
Tendencias de la segregación residencial en León, Guanajuato (1990-2010)

CUADRo 1. Variables incluidas en el análisis factorial por componentes principales

\begin{tabular}{lccc}
\multicolumn{1}{c}{ Variables } & 1990 & 2000 & 2010 \\
\hline $\begin{array}{l}\text { Grado promedio de escolaridad } \\
\text { Población ocupada de la PEA (\%) }\end{array}$ & $\mathrm{X}$ & $\mathrm{X}$ & $\mathrm{X}$ \\
$\begin{array}{l}\text { Población con derechohabiencia a servicios de salud } \\
\text { pública o privada (\%) }\end{array}$ & $\mathrm{X}$ & $\mathrm{X}$ \\
$\begin{array}{l}\text { Promedio de ocupantes por cuarto en las viviendas } \\
\text { Viviendas con piso de material diferente a tierra (\%) }\end{array}$ & $\mathrm{X}$ & $\mathrm{X}$ & $\mathrm{X}$ \\
$\begin{array}{l}\text { Viviendas que disponen de drenaje (\%) } \\
\text { Viviendas que disponen de agua entubada en el }\end{array}$ & $\mathrm{X}$ & $\mathrm{X}$ & $\mathrm{X}$ \\
$\begin{array}{l}\text { ámbito de la vivienda (\%) } \\
\text { Población de 15 años y más con instrucción } \\
\text { postprimaria (\%) }\end{array}$ & $\mathrm{X}$ & $\mathrm{X}$ \\
Viviendas con más de un dormitorio (\%) & & $\mathrm{X}$ & \\
PEa ocupada con ingresos de más de 2 SMM (\%) & $\mathrm{X}$ & & \\
\hline
\end{tabular}

Fuente: Elaboración propia

sobre el comportamiento de sus distribuciones (el cuadro 1 señala las variables incluidas en el modelo para cada año). Estas variables se definieron inicialmente para el año más reciente del análisis (2010); para el 2000 se contó con las siete variables a excepción de un ajuste en la variable del material de los pisos de las viviendas, mientras que para 1990 fue necesario incluir tres variables proxy.

Este periodo de análisis no solo coincide con la disponibilidad de información censal, sino también con el inicio de las manifestaciones del cambio de modelo económico y de producción, que en la región latinoamericana ha estado modificando el esquema socioespacial de las ciudades (Sassen, 1991; Schteingart, 2010). De manera que a partir de los análisis por AGEB es posible observar las tendencias de la segregación durante la última etapa del desarrollo urbano de la ciudad de León (García, 2010) y en un periodo que comprende grandes cambios sociales, económicos y urbanos en el país y en la región latinoamericana. 


\section{Análisis por AGEB en 1990}

Como se aprecia en el cuadro 2, para 1990 la ciudad de León estaba conformada por siete localidades urbanas y 796775 habitantes, de los cuales $95 \%$ se encontraba en la localidad de León de los Aldama. El total de ageb urbanas es de 204, también con una amplia concentración en la principal localidad urbana (87 \% pertenece a León de los Aldama). De las siete localidades, dos contaban con un ageb y una con dos ageb; de igual modo, solo dos localidades superan los 10000 habitantes.

CuAdro 2. Población y AGEB por localidades de la Ciudad de León 1990

\begin{tabular}{|c|c|c|c|c|}
\hline Localidad & Población & Porcentaje & AGEB & Porcentaje \\
\hline Los Castillos & 12683 & 1.6 & 8 & 3.9 \\
\hline $\begin{array}{l}\text { Centro Familiar la } \\
\text { Soledad }\end{array}$ & 3930 & 0.5 & 5 & 2.5 \\
\hline Duarte & 5545 & 0.7 & 1 & 0.5 \\
\hline $\begin{array}{l}\text { Fraccionamiento } \\
10 \text { de Mayo }\end{array}$ & 10100 & 1.3 & 9 & 4.4 \\
\hline León de los Aldama & 758279 & 95.2 & 178 & 87.3 \\
\hline $\begin{array}{l}\text { Plan de Ayala (Santa } \\
\text { Rosa) }\end{array}$ & 3673 & 0.5 & 1 & 0.5 \\
\hline Presitas & 2565 & 0.3 & 2 & 1.0 \\
\hline Total & 796775 & 100.0 & 204 & 100.0 \\
\hline
\end{tabular}

Fuente: Elaboración propia con base en el Censo General de Población y Vivienda 1990 (INEGI, en línea).

De las variables clave seleccionadas para el análisis factorial de 2010, solo cuatro se pueden replicar exactamente para 1990, pues en este censo aún no se tenían en cuenta algunos temas que se han ido incorporando a través de los eventos censales de acuerdo con la agenda pública y de investigación. Así, se han calculado tres indicadores proxy con similar temática, capacidad de explicación y diferenciación socioespacial. De esta manera, se ha realizado el análisis de componentes principales con las variables del cuadro 3 , del cual resulta un componente principal (Factor I) que explica $67 \%$ de la varianza total, lo que para efectos de este trabajo se considera como un buen estimador. 
Las variables con mayor ponderación en el Factor I son: número de dormitorios, agua potable, escolaridad, materiales de la vivienda y drenaje. Como se observa, de las cinco principales, cuatro corresponden a características físicomateriales de la vivienda, por lo que se puede decir que el aspecto primordial para la diferenciación socioespacial en la década de 1990 lo constituyen las condiciones materiales de las viviendas y no tanto las características sociodemográficas de la población.

CUAdRo 3. Factor extraído del análisis de componentes principales 1990

\section{Variables}

Porcentaje de población ocupada de la PEA (ocupados)

Porcentaje de viviendas con piso de material diferente a tierra (piso)

Porcentaje de viviendas que disponen de drenaje (drenaje)

Porcentaje de viviendas con agua potable dentro de la vivienda (agua)

Porcentaje de población de 15 años y más con instrucción postprimaria (escolaridad)

Porcentaje de viviendas con más de 1 dormitorio (dormitorios)

Porcentaje de la PEA ocupada con ingresos de más de 2 SMM (ingresos)

Método de extracción: Análisis de componentes principales; 1 componente extraído Fuente: Elaboración propia

Los valores que toma el Factor I se han agrupado en cinco estratos a partir de su distribución: muy bajo (menos de -2.000), bajo (de -2.000 a -1.001), medio (de -1.000 a -0.001), medio alto (de 0.000 a 0.999 ) y alto (de 1.000 a 1.999), siendo los mismos para los tres momentos analizados, por lo que se asegura la comparabilidad entre cada año.

Cada estrato socioespacial representa un sector de la población homogéneo entre sí, pero lo más distinto posible de los estratos superior e inferior; esta es una de las cualidades del análisis factorial por componentes principales y la razón principal para su elección como técnica de análisis. Con ello se busca la mayor homogeneidad de cada estrato y al mismo tiempo una diferenciación de los demás. El análisis factorial se pudo realizar para un total de 177 AGEB pues 27 no contaron con información suficiente; estas AGEB representan $87 \%$, 
pero las que se quedaron fuera son las menos pobladas ya que solo constituyen $2.3 \%$ de la población total.

De tal suerte, en el cuadro 4 se observa que la mayor proporción de las AGEB se ubica en el estrato medio alto, concentrando a seis de cada diez habitantes de la ciudad; el segundo estrato en importancia es el medio, con $20 \%$ de las AGEB pero la cuarta parte de la población.

La relación de población por AGEB deja ver que los estratos de los extremos (muy bajo, bajo y alto) son los menos poblados, principalmente los primeros dos. Si se tiene en cuenta que la ponderación de las variables es mayor en lo físicomaterial, entonces se puede inferir que estos estratos también pueden caracterizarse por una reciente formación, probablemente localizados en las periferias de la zona urbana. Para el caso de los estratos inferiores, estos podrían corresponder a las nuevas expansiones urbanas informales, en tanto que el estrato alto respondería a los nuevos fraccionamientos cerrados que no pasan por un proceso de consolidación, pues desde el comienzo cuentan con todos los servicios urbanos, pero inicialmente se encuentran poco poblados.

CUADRO 4. AGEB y población por estratos socioespaciales 1990

\begin{tabular}{|c|c|c|c|c|c|}
\hline Estratos & AGEB & Porcentaje & Población & Porcentaje & Pob./ ageb \\
\hline Muy bajo & 10 & 5.6 & 13651 & 1.8 & 136510 \\
\hline Bajo & 20 & 11.3 & 38814 & 5.0 & 194070 \\
\hline Medio & 36 & 20.3 & 191233 & 24.6 & 531203 \\
\hline $\begin{array}{l}\text { Medio } \\
\text { alto }\end{array}$ & 94 & 53.1 & 483753 & 62.1 & 514631 \\
\hline Alto & 17 & 9.6 & 51174 & 6.6 & 301024 \\
\hline Total & 177 & 100.0 & 778625 & 100.0 & 439901 \\
\hline
\end{tabular}

Fuente: Elaboración propia.

Debido a que las variables seleccionadas tienen amplia relación con la estructura social de la ciudad, los estratos también representan una jerarquía, como lo expresan los valores del cuadro 5 en donde el estrato muy bajo tiene los promedios más bajos de la estratificación socioespacial. Esta situación refuerza el argumento anterior, con relación a que los estratos muy bajo y bajo concentran las nuevas expansiones urbanas en proceso de consolidación, 
muy probablemente a los asentamientos informales que aún no cuentan con la infraestructura que provee a las viviendas de los servicios básicos de agua potable, drenaje y electricidad.

Según se advierte en el cuadro 5, las mayores distancias socioeconómicas que impactan sobremanera en las distancias socioespaciales son el drenaje, el agua potable y la escolaridad, pues es donde las varianzas de los valores promedio presentan los rangos más amplios entre el estrato muy bajo y alto. La ocupación y el ingreso constituyen aspectos de menor diferenciación, lo que también indica que la población de los estratos bajos no necesariamente concentra a toda la población de menores ingresos ni marginada del mercado laboral, sino que al existir una mayor heterogeneidad en estas variables se confirma la noción de que la estructura espacial no es un reflejo de la estructura social, sino que en la primera intervienen además otros factores, que en este caso pudiera ser la falta de acceso a la seguridad social que limita el acceso al mercado formal, y que aun teniendo un poder adquisitivo con el cual calificar para un financiamiento a la vivienda, no es posible hacerlo por los requisitos de los programas públicos para vivienda.

Por otro lado, el acelerado crecimiento de la población en estas décadas ha rebasado la capacidad del gobierno y del sector privado formal para incorporar suelo urbano y producir vivienda en esquemas formales, lo que ha derivado en la incorporación de suelo informal al mercado habitacional urbano, configurándose en muchos casos la única opción para grandes sectores de la población, más allá de su ubicación en la jerarquía social de la ciudad.

CUAdRo 5. Valores medios de las variables por estratos socioespaciales 1990

\begin{tabular}{lrcccccc}
\multicolumn{1}{c}{ Estratos } & Escolaridad & Ocupados & Ingresos & Dormitorios & Piso & Drenaje & Agua \\
Muy bajo & 9.9 & 95.6 & 43.1 & 44.8 & 68.7 & 5.7 & 7.0 \\
Bajo & 14.9 & 97.7 & 44.1 & 53.3 & 72.5 & 43.2 & 40.3 \\
Medio & 27.6 & 97.8 & 47.7 & 72.6 & 90.6 & 86.0 & 73.5 \\
Medio alto & 48.5 & 98.4 & 55.1 & 83.8 & 96.3 & 94.3 & 88.3 \\
Alto & 79.6 & 98.9 & 67.8 & 90.6 & 97.2 & 97.1 & 95.1 \\
\multicolumn{1}{r}{ Total } & 41.7 & 98.1 & 52.8 & 76.5 & 91.0 & 82.1 & 76.0
\end{tabular}




\section{Análisis por AGEB en 2000}

Para el año 2000 la ciudad de León está constituida por 362 AGEB con 1065981 habitantes, distribuidos en ocho localidades; la concentración en la localidad de León de los Aldama se conserva similar a la década anterior. De las siete localidades de 1990, solo se mantienen cuatro en 2000, pero con la aparición de cuatro nuevas que hasta hace poco tiempo eran consideradas localidades rurales.

CuAdro 6. Población y ageb por localidades de la ciudad de León 2000

\begin{tabular}{|c|c|c|c|c|}
\hline Localidad & Población & Porcentaje & AGEB & Porcentaje \\
\hline $\begin{array}{l}\text { Álvaro Obregón } \\
\text { (Santa Ana del } \\
\text { Conde) }\end{array}$ & 2683 & 0.3 & 1 & 0.3 \\
\hline $\begin{array}{l}\text { Centro Familiar La } \\
\text { Soledad }\end{array}$ & 18526 & 1.7 & 16 & 4.4 \\
\hline Duarte & 5671 & 0.5 & 3 & 0.8 \\
\hline La Ermita & 4802 & 0.5 & 4 & 1.1 \\
\hline León de Los Aldama & 1020818 & 95.8 & 329 & 90.9 \\
\hline $\begin{array}{l}\text { Loza de los Padres } \\
\text { (La Loza) }\end{array}$ & 2290 & 0.2 & 1 & 0.3 \\
\hline Medina & 6648 & 0.6 & 7 & 1.9 \\
\hline \multirow[t]{2}{*}{$\begin{array}{l}\text { Plan de Ayala } \\
\text { (Santa Rosa) }\end{array}$} & 4543 & 0.4 & 1 & 0.3 \\
\hline & 1065981 & 100.0 & 362 & 100.0 \\
\hline
\end{tabular}

Fuente: Elaboración propia

El análisis factorial para este año se realiza con las siete variables originales (cuadro 7). El método de análisis de componentes principales extrae dos componentes que agrupan $73.5 \%$ de la varianza total; el primer componente (Factor I) agrupa $58.2 \%$, mientras que el segundo (Factor II), $15.3 \%$. En el Factor I, las variables con mayor ponderación son las referentes al agua potable, ocupantes por vivienda, pisos, drenaje y escolaridad; en el Factor II, la variable con mayor ponderación es la ocupación de la población en el mercado de trabajo. Estos datos indican que las condiciones materiales de la vivienda siguen siendo los aspectos más relevantes para la estructura socioespacial de la ciudad. 
CUAdRo 7. Factor extraído del análisis de componentes principales 2000

\begin{tabular}{|c|c|c|}
\hline Variables & Factor I & Factor II \\
\hline Grado promedio de escolaridad (escolaridad) & 0.800 & 0.271 \\
\hline Porcentaje de población ocupada (ocupados) & 0.039 & 0.967 \\
\hline $\begin{array}{l}\text { Porcentaje de población derechohabiente a servicios de } \\
\text { salud (salud) }\end{array}$ & 0.689 & -0.044 \\
\hline $\begin{array}{l}\text { Promedio de ocupantes por cuarto en viviendas } \\
\text { (hacinamiento) }\end{array}$ & -0.885 & -0.095 \\
\hline $\begin{array}{l}\text { Porcentaje de viviendas con piso de cemento, madera, } \\
\text { mosaico y otro (piso) }\end{array}$ & 0.832 & -0.137 \\
\hline $\begin{array}{l}\text { Porcentaje de viviendas con drenaje conectado a la red } \\
\text { pública (drenaje) }\end{array}$ & 0.825 & -0.160 \\
\hline $\begin{array}{l}\text { Porcentaje de viviendas con agua potable dentro de la } \\
\text { vivienda (agua) }\end{array}$ & 0.896 & -0.069 \\
\hline
\end{tabular}

Fuente: Elaboración propia

Por la disponibilidad de información, el análisis factorial pudo efectuarse para 346 AGEB (cuadro 8), que representan $96 \%$ del total pero concentran $99 \%$ de la población total, pues los 16 AGEB sin información suficiente solo agrupan a 147 personas. De estas 346 AGEB y con los valores del Factor I, más de la mitad se ubican en el estrato medio alto, que también es el estrato con mayor población en promedio por AGEB y en conjunto representan cerca de las tres cuartas partes de la población total. El segundo estrato en importancia por el número de AGEB es el medio con $20 \%$, aunque con menor proporción de población (17 \%), y constituye el tercer lugar en la tasa de población por AGEB, a diferencia de 1990 cuando era el estrato con la tasa más alta, lo cual indica un proceso de despoblamiento notable en las AGEB de este estrato. Le siguen en proporción poblacional los estratos alto y bajo; finalmente, el estrato muy bajo presenta una tasa de población por AGEB muy baja, y aunque agrupa a $6 \%$ de las AGEB, no alcanza $1 \%$ de población.

Como se observó en la ponderación de las variables para cada factor, la dimensión físico-material de las viviendas es la que mayor diferenciación socioespacial aporta. 
CuAdro 8. Ageb y población por estratos socioespaciales 2000

\begin{tabular}{l|rr|rr|r}
\multicolumn{1}{c}{ Estratos } & AGEB & Porcentaje & Población & Porcentaje & Pob./ AGEB \\
\hline Muy bajo & 21 & 6.1 & 3155 & 0 & 150 \\
Bajo & 39 & 11.3 & 57308 & 5 & 1469 \\
Medio & 70 & 20.2 & 176208 & 17 & 2517 \\
Medio alto & 195 & 56.4 & 774110 & 73 & 3970 \\
Alto & 21 & 6.1 & 55053 & 5 & 2622 \\
\multicolumn{1}{r}{ Total } & 346 & 100.0 & 1065834 & 100 & 3080 \\
\hline
\end{tabular}

Fuente: Elaboración propia

Esto se verifica en el cuadro 9 sobre todo con la variable de drenaje, en la cual se presenta el rango más amplio entre los valores medios del estrato muy bajo y alto, pues mientras en el alto prácticamente hay una cobertura total, en el muy bajo esta es de solo $6 \%$; la segunda variable con el rango más amplio es el agua potable con similar diferenciación entre los estratos inferior y superior. La escolaridad de la población resulta la variable socioeconómica con mayores diferencias entre estratos, e incluso el estrato medio exhibe un valor promedio inferior a seis grados de escolaridad, lo que constituye menos de lo necesario para concluir la primaria; el promedio de grados de escolaridad del estrato alto representa cerca de dos años de bachillerato.

CuAdro 9. Valores medios de las variables por estratos socioespaciales 2000

\begin{tabular}{lccccccc}
\multicolumn{1}{c}{ Estratos } & Escolaridad & Ocupados & Salud & Hacinamiento & Piso & Drenaje & Agua \\
Muy bajo & 4.0 & 99.9 & 30.3 & 3.3 & 55.2 & 6.2 & 5.8 \\
Bajo & 4.7 & 98.6 & 40.1 & 2.7 & 80.6 & 31.2 & 18.0 \\
Medio & 5.4 & 98.9 & 49.6 & 2.1 & 91.0 & 80.9 & 56.5 \\
Medio alto & 8.3 & 99.1 & 57.4 & 1.4 & 98.1 & 97.0 & 92.6 \\
Alto & 10.9 & 99.3 & 73.2 & 1.0 & 99.1 & 99.0 & 97.1 \\
\multicolumn{1}{r}{ Total } & 7.2 & 99.1 & 53.2 & 1.8 & 92.1 & 80.9 & 71.9
\end{tabular}

Fuente: Elaboración propia

La distribución espacial de los estratos en León (figura 1) muestra todavía la estructura de grandes zonas de la ciudad, pero con algunas AGEB de estrato 
medio en zonas principalmente ocupadas por estratos bajos y medio-altos, lo que ejemplifica el proceso de la reducción de la escala en la observación de la segregación, pues las grandes zonas tradicionales de la ciudad van adquiriendo una estratificación mayor para el año 2000.

Los estratos muy bajo y bajo exhiben una localización más definida, sobre todo el estrato muy bajo que prácticamente se ubica en las periferias de la ciudad, y aunque también el estrato bajo se sitúa en ellas, al igual se encuentra en otras zonas de la ciudad.

Los estratos altos, en cambio, se hallan más concentrados en dos zonas de la ciudad -el norte y el sur-, las AGEB están muy próximas entre sí y tienen alrededor principalmente AGEB de estratos medio-altos, lo que indica que aun para el año 2000 las zonas de estrato alto presentan una alta concentración en la ciudad de León y al mismo tiempo están relativamente aisladas de los estratos más bajos.

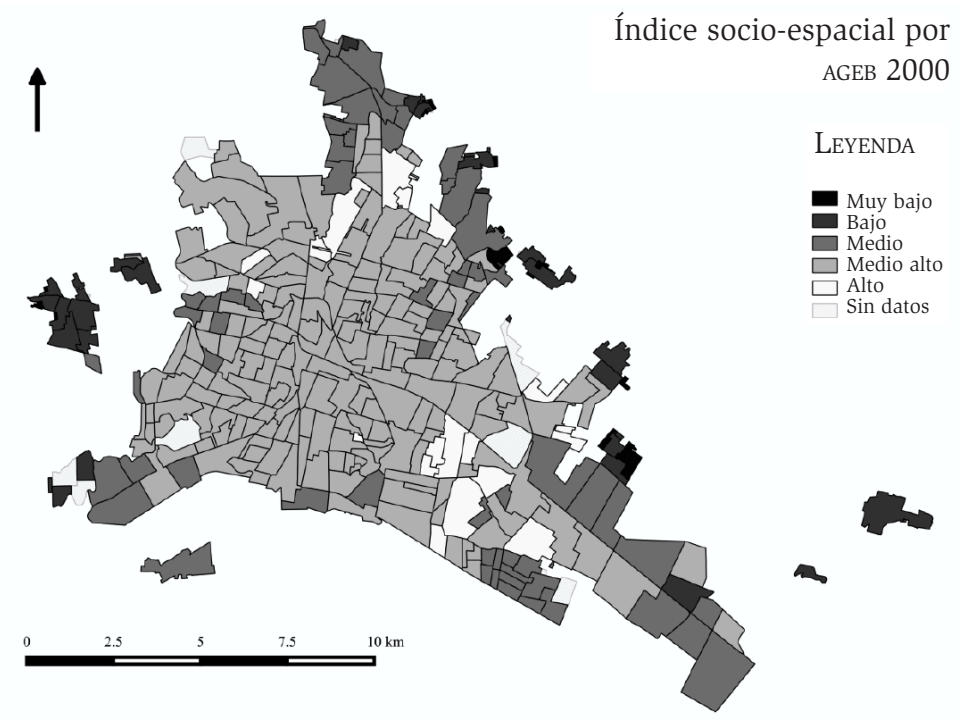

Fuente: Elaboración propia 


\section{Análisis por AGEB en 2010}

Para 2010 León está configurada por 12 localidades urbanas y 1337570 personas. Integran la ciudad 552 AGEB que se distribuyen de acuerdo al cuadro 10. Para este año no hubo cambios en las localidades del 2000 pues las ocho de ese año se mantienen, aunque hubo un incremento de cuatro localidades que durante esta década se conformaron como urbanas y pasaron a formar parte de la ciudad de León.

El crecimiento más importante después de León de los Aldama se registra en La Ermita, que cuadruplicó su población del 2000, seguido del Centro Familiar la Soledad, que casi la duplicó. Durante esta década Medina aumenta en 250 \% su población, pero el crecimiento más relevante en términos absolutos se da hacia las localidades ya mencionadas.

CuAdRo 10. Población y AGEB por localidades de la ciudad de León 2010

\begin{tabular}{|c|c|c|c|c|}
\hline Localidad & Población & Porcentaje & AGEB & Porcentaje \\
\hline $\begin{array}{l}\text { Álvaro Obregón } \\
\text { (Santa Ana del Conde) }\end{array}$ & 3456 & 0.3 & 2 & 0.4 \\
\hline $\begin{array}{l}\text { Centro Familiar la } \\
\text { Soledad }\end{array}$ & 32159 & 2.4 & 31 & 5.6 \\
\hline Duarte & 6261 & 0.5 & 13 & 2.4 \\
\hline La Ermita & 19703 & 1.5 & 13 & 2.4 \\
\hline León de los Aldama & 1238962 & 92.6 & 457 & 82.8 \\
\hline Loza de los Padres & 2875 & 0.2 & 1 & 0.2 \\
\hline Medina & 16166 & 1.2 & 24 & 4.3 \\
\hline $\begin{array}{l}\text { Plan de Ayala } \\
\text { (Santa Rosa) }\end{array}$ & 5134 & 0.4 & 1 & 0.2 \\
\hline $\begin{array}{l}\text { Rizos de la Joya } \\
\text { (Rizos del Saucillo) }\end{array}$ & 2694 & 0.2 & 2 & 0.4 \\
\hline San Juan de Abajo & 4514 & 0.3 & 2 & 0.4 \\
\hline San Juan de Otates & 2905 & 0.2 & 5 & 0.9 \\
\hline $\begin{array}{l}\text { San Nicolás de los } \\
\text { González }\end{array}$ & 2741 & 0.2 & 1 & 0.2 \\
\hline Total & 1337570 & 100.0 & 552 & 100.0 \\
\hline
\end{tabular}

Fuente: Elaboración propia. 
El análisis de componentes principales arrojó un factor (Factor I) que explica $51.1 \%$ de la varianza total; las variables con alta ponderación son la disponibilidad de agua dentro de la vivienda, el promedio de ocupantes por cuarto, el grado de escolaridad y el material del piso de la vivienda (cuadro 8). De las siete variables incluidas, cuatro están relacionadas con las condiciones de la vivienda y tres con las de las personas y, como hemos visto, las primeras tienen mayor ponderación en la determinación del índice de segregación socioespacial que se ha construido.

CuADRo 11. Factor extraído del análisis de componentes principales 2010

Variables

Grado promedio de escolaridad (escolaridad)

Porcentaje de población ocupada de la PEA (ocupados)

Porcentaje de población con derechohabiencia a servicios de salud (salud)

Promedio de ocupantes por cuarto en viviendas (hacinamiento)

Porcentaje de viviendas con piso de material diferente a tierra (piso)

Porcentaje de viviendas que disponen de drenaje (drenaje)

Porcentaje de viviendas con agua potable dentro de la vivienda (agua)

Método de extracción: Análisis de componentes principales. 1 componente extraído Fuente: Elaboración propia
Factor I

0.792

0.538

0.767

0.675

0.829

La disponibilidad de información permitió calcular el índice socioespacial para 489 AGEB; las 63 AGEB para las que no pudo calcularse el Factor I represen$\tan 11 \%$ de las AGEB pero solo $0.4 \%$ de la población total de la ciudad.

En el cuadro 12 se aprecia que el estrato que concentra el mayor número de AGEB y población es el medio alto con 56 y $72 \%$, respectivamente, seguido del estrato medio con $20 \%$ de las AGEB y $18 \%$ de la población; los estratos bajo y alto presentan la misma frecuencia en AGEB ( 45 cada uno) aunque no en población, ya que las AGEB de estrato alto son en promedio más pobladas que las de estrato bajo, con 1752 y 951 habitantes, respectivamente. 
CUAdRo 12. Ageb y población por estratos socioespaciales 2010

$\begin{array}{lrrrrr}\text { Estratos } & \text { AGEB } & \text { Porcentaje } & \text { Población } & \text { Porcentaje } & \text { Pob./ AGEB } \\ \text { Muy bajo } & 26 & 5.3 & 5527 & 0.4 & 213 \\ \text { Bajo } & 45 & 9.2 & 42807 & 3.2 & 951 \\ \begin{array}{l}\text { Medio } \\ \text { Medio }\end{array} & 100 & 20.4 & 244434 & 18.4 & 2444 \\ \text { alto } & 273 & 55.8 & 960254 & 72.1 & 3517 \\ \text { Alto } & 45 & 9.2 & 78819 & 5.9 & 1752 \\ \quad \text { Total } & 489 & 100 & 1331841 & 100 & 2724\end{array}$

Fuente: Elaboración propia

Si se tiene en cuenta que las AGEB del estrato muy bajo son todavía menos pobladas, además de la mayor ponderación de las variables relacionadas con las condiciones físicas de las viviendas, se tienen dos elementos que hacen referencia a la consolidación urbana como elemento clave para entender la segregación en los estratos bajos. Una baja población indica -no en todos los casos pero al menos sí en esta reflexión- recientes procesos de urbanización y donde los asentamientos aún no se encuentran consolidados como lo están las zonas más pobladas de la ciudad.

Las diferencias en las condiciones de vida de la población, de acuerdo con los estratos socioespaciales que se han construido, son evidentes y muy amplias entre los estratos más bajos y más altos, como se puede observar en el cuadro 13. En promedio, la población de los estratos muy bajo y bajo no concluye la educación primaria (se necesitarían seis grados de escolaridad), en tanto que la población del estrato alto tiene en promedio estudios de nivel superior (más de los doce años requeridos para preparatoria). Estas diferencias en la escolaridad también se manifiestan en la ocupación de la población económicamente activa, pues el desempleo es mayor en los estratos bajos, mientras que a medida que aumenta el nivel del estrato disminuye el desempleo.

En general, las condiciones del estrato muy bajo son inferiores a las de los demás estratos, sin embargo, la mayor diferencia se presenta en la variable de la disponibilidad de agua potable dentro de la vivienda, en la cual se manifiestan dos situaciones: por un lado, la disponibilidad de la infraestructura (red pública de agua potable) y, por otro, la capacidad de las familias respecto a instalar las conexiones necesarias para introducirla al interior de la vivienda. Como resultado, 
la disponibilidad de agua al interior de la vivienda para el estrato muy bajo es de solo $3 \%$ de las viviendas, mientras que el siguiente estrato ya alcanza $20 \%$ y el medio está cerca de $80 \%$; finalmente, en el estrato alto solo $2 \%$ de las viviendas no cuenta con esta conexión domiciliaria.

CUADRo 13. Valores medios de las variables por estratos socioespaciales 2010

\begin{tabular}{lrrrrrrr}
\multicolumn{1}{c}{ Estratos } & Escolaridad & Ocupados & Salud & Hacinamiento & Piso & Drenaje & Agua \\
Muy bajo & 5.0 & 92.4 & 59.6 & 2.1 & 74.5 & 54.8 & 2.8 \\
Bajo & 5.9 & 93.3 & 66.3 & 1.7 & 86.0 & 86.5 & 20.2 \\
Medio & 6.7 & 94.7 & 68.0 & 1.4 & 92.9 & 94.5 & 79.2 \\
Medio alto & 9.3 & 96.1 & 70.9 & 1.0 & 97.0 & 98.1 & 96.2 \\
Alto & 13.2 & 98.0 & 77.2 & 0.7 & 97.0 & 97.9 & 97.7 \\
$\quad$ Total & 8.6 & 95.5 & 69.9 & 1.2 & 94.0 & 94.0 & 80.9
\end{tabular}

Fuente: Elaboración propia

La figura 2 representa la distribución espacial de los estratos en 2010 y muestra una ciudad donde las grandes zonas urbanas se van diluyendo cada vez más, sobre todo aquellas tradicionalmente identificadas como de estratos altos, pues aunque la mayoría de estas AGEB mantiene esta ubicación, otras AGEB que alcanzaron esta condición ya no se encuentran tan próximas a las que existían en el 2000, en cambio, las nuevas AGEB de estratos altos se localizan en zonas antes identificadas como de estratos medio-altos y medios.

También las AGEB de estrato bajo se ubican en zonas más amplias de la ciudad e incluso algunas se hallan en zonas no tradicionales. Sin embargo, es el estrato medio el que presenta una distribución más amplia por toda la mancha urbana, y si bien no constituye el estrato con mayor número de AGEB, sí es el que se encuentra más próximo a todos los demás estratos socioespaciales en el espacio urbano. 


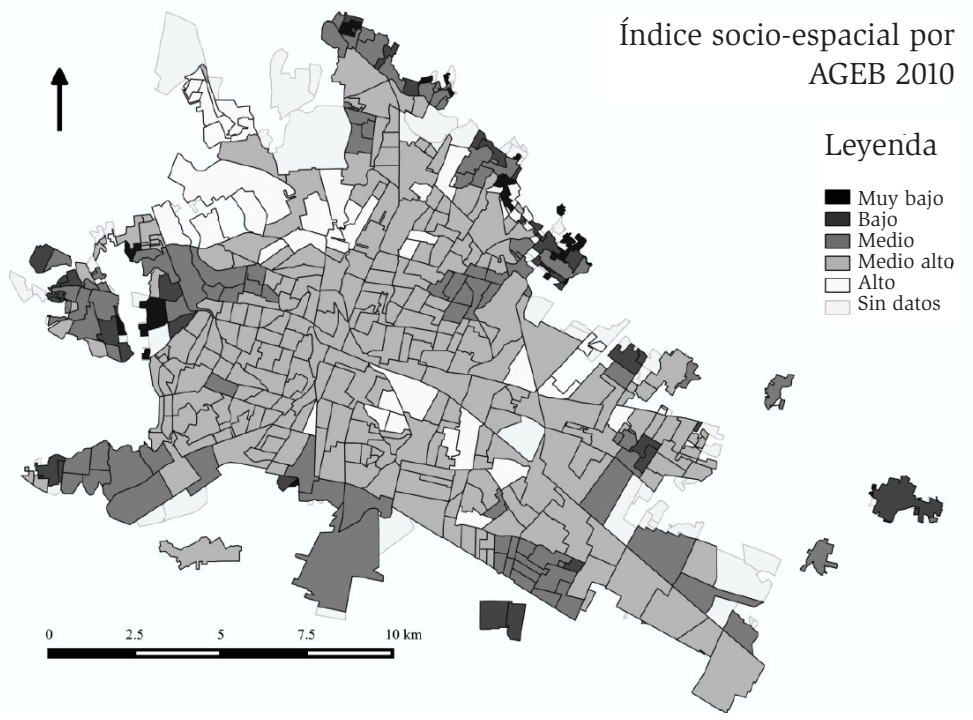

Fuente: Elaboración propia

Figura 2. Estratificación socioespacial de la ciudad de León 2010

\section{Discusión}

El análisis de la segregación objetiva de las últimas dos décadas tiene relevancia ya que, de acuerdo con Castells (1976), una ciudad no solo expresa la estructura social presente, sino que en cada caso combina, en un momento dado, las expresiones de varias estructuras sociales. Si bien la estructura social es solamente un aspecto que configura la segregación, para entenderla en su situación actual se hace necesario revisar los procesos urbanos a través del tiempo, lo que se ha intentado hacer en este estudio, aunque limitado a la comparación de tres momentos (1990, 2000 y 2010) por la disponibilidad de información estadística.

El primer referente encontrado acerca de la segregación en la ciudad de León es en el trabajo de García (2010), que menciona la construcción del libramiento norte hacía finales de la década de 1970 como una vialidad que provocó una expansión hacia la zona nororiente, generando altos niveles de diferenciación y segregación. Asumiendo que el fenómeno de la segregación se intensificara durante la década de 1980, fue en el censo de 1990 cuando por primera vez 
se pudo observar de manera más evidente en la estructura socioespacial de la ciudad. Antes de ello, la división social del espacio de la ciudad se presentaba en grandes zonas urbanas, con el centro histórico como principal referente y con la aparición de algunos subcentros urbanos, relacionado con el modelo de núcleos múltiples en las ciudades como producto de las transformaciones urbanas a partir de los años cuarenta.

El primer aspecto que sobresale en el análisis de estas últimas dos décadas es la acelerada expansión de la mancha urbana y de la población, pues la ciudad casi duplicó su población al mismo tiempo que fue aumentando el número de AGEB, tanto por el crecimiento demográfico de su principal localidad, León de los Aldama, como por el crecimiento e incorporación a la ciudad de localidades más pequeñas, consideradas rurales hasta pocos años anteriores a 1990. La disminución de la tasa de población por AGEB en todos los estratos del análisis muestra que la ciudad no solo se ha extendido en su mancha urbana, sino también que ha habido una subdivisión importante de AGEB; este hecho confirma para la ciudad de León una de las dos principales tendencias de la segregación residencial: una diferenciación socioespacial a menor escala (Sabatini, Cáceres y Cerda, 2001; Aguilar y Mateos, 2011).

Sobre la dispersión urbana, esta se verificó a partir de la disminución del promedio de habitantes por AGEB; la población total de la ciudad aumentó en 71 \% y el número de AGEB lo hizo en 176 \% , lo cual implicó que la densidad por AGEB haya disminuido de 4399 habitantes en 1990 a 2724 en 2010. No se encontraron indicios de cambios en esta tendencia en el corto o mediano plazo, por lo que es posible inferir que la dispersión urbana se mantendrá como una característica de la ciudad actual, tanto por la integración de nuevo suelo urbano a la ciudad, como por la incorporación de localidades hasta ahora consideradas rurales pero que en próximos años alcanzarán su condición de urbanas por propio crecimiento, o bien serán absorbidas por la mancha urbana de la ciudad. Es pertinente recordar que en 1990 la ciudad se conformaba por siete localidades urbanas, en 2000 aumentó a ocho y en 2010 fueron 12 las localidades urbanas del municipio de León. El último censo de población registra 29 localidades de entre 1000 y 2499 habitantes, lo que hace previsible que durante los próximos años varias de ellas alcancen la categoría de urbanas y se integren a la ciudad de León. Lo que se ha visto con este tipo de localidades es que al incorporarse a la ciudad pasan a formar parte de las periferias urbanas con amplias carencias 
en la infraestructura y con altas probabilidades de alojar a los sectores populares de la población en condiciones de segregación socioespacial relacionadas con los estratos bajos.

Continuar con el modelo de expansión y dispersión urbana sin que se modifiquen las condiciones estructurales de la misma provoca que las tendencias de la segregación se mantengan y que, como plantea Harvey (1992), estos procesos de diferenciación reproduzcan las desigualdades sociales. Ya sea a través de la urbanización popular o de fraccionamientos cerrados, la expansión de la ciudad estará caracterizada por una intensificación de la diferenciación socioespacial, lo que conduce a la siguiente tendencia de la segregación para la ciudad de León.

Cuando se menciona que la escala de observación de la segregación está reduciéndose, no es únicamente por la posibilidad de realizar análisis intraurbanos con base en la desagregación de la información censal en las AGEB, sino también porque a partir de la definición de estratos socioespaciales para los momentos censales la distribución espacial de estos estratos en la ciudad está teniendo un comportamiento específico que se definió como una tendencia de la segregación. Aunque no se dispone de información por AGEB anterior a 1990, lo cual imposibilita efectuar análisis intraurbanos similares, la información recopilada acerca de la división social del espacio en León anterior a 1990 habla de una ciudad con grandes zonas muy bien identificadas por su homogeneidad social.

El centro histórico, las zonas de desarrollo habitacional para estratos altos, las de crecimiento irregular, las industriales y otras, habían sido parte del imaginario colectivo en torno a la división de la ciudad. Pero lo que se observa a partir de 1990 es una dispersión de las AGEB de estrato alto hacia otras áreas de la ciudad, ya sean nuevas u ocupadas por distintos estratos; de la misma forma, los estratos medio alto y medio no solo han incrementado su proporción respecto al total, sino que se localizan en prácticamente todas las zonas de la ciudad; caso contrario es el de los estratos más bajos (bajo y muy bajo), que han reducido ligeramente su proporción, y cada vez se encuentran más concentrados en algunas áreas muy específicas de la ciudad, sobre todo en las periferias urbanas del oriente y poniente.

Esto no necesariamente se trata de un desplazamiento de unos grupos sociales por otros en los términos de Puebla (2007), sino que aunque en efecto 
algunas zonas recientemente revalorizadas a partir de intervenciones urbanas están siendo ocupadas por estratos medios o altos desplazando a los estratos bajos, también y en mayor medida zonas antes consideradas de estrato medio tienen una movilidad hacia el medio-alto a raíz de la consolidación de la ciudad, manteniendo preponderantemente a la misma población; de hecho, para 2010, la zona de "alta renta" (Sabatini, 2003) es cada vez más evidente a partir de la distribución del estrato alto en los mapas que se han realizado. Sin embargo, esta situación no está ocurriendo con los estratos bajos y muy bajos, ya que la movilidad en la estratificación socioespacial es muy reducida e incluso incorpora a las nuevas zonas de urbanización popular; esto se constata, ya que en 1990 el número de AGEB de los estratos muy bajo y bajo eran 30, para el año 2000 aumentaron a 60 y para 2010 a 71.

La dispersión de los estratos medios, medio-altos y altos hacia muchas más zonas de la ciudad se ha identificado como un acercamiento espacial entre los diferentes estratos, pero que no necesariamente implica una interacción social, pues, aunque no es motivo del presente trabajo, se observa que este acercamiento espacial se da principalmente a través de fraccionamientos cerrados que se localizan en suelo de menor valor, autosegregándose de su entorno inmediato. Los mapas de la estratificación socioespacial para 2000 y 2010 son muy claros en este sentido.

La tercera tendencia de la segregación para la ciudad de León se trata del aumento de la diferenciación entre los estratos socioespaciales y corresponde con la intensificación de los efectos negativos de la segregación; se observó a partir del incremento de las diferencias de los valores medios de las variables incluidas en la definición de los estratos socioespaciales. En términos generales, la producción y reproducción del espacio residencial de la ciudad transcurre por un proceso de consolidación, con mayores niveles de cobertura en infraestructura de servicios básicos, vialidades y equipamiento, entre otros. Sin embargo, los beneficios de esta consolidación no se distribuyen igualitariamente, antes bien, las diferencias encontradas entre los estratos socioespaciales en 1990 han aumentado con el tiempo.

Aunque las proporciones de AGEB y de población en los estratos muy bajos y bajos han ido disminuyendo, las condiciones de vida de esta población no están mejorando o lo hacen de manera más lenta que en el resto de la ciudad. En las últimas décadas algunos sectores de la ciudad han logrado una movilidad 
ascendente entre los estratos por su proceso de consolidación, sobre todo por la introducción de redes de infraestructura pública, la mejoría de las viviendas y el aumento de la escolaridad de sus habitantes, lo cual se observa sobre todo en los estratos medios que han pasado a considerarse como medio-altos; empero, existen zonas de la ciudad que no logran una mejoría sustancial e incluso en algunos aspectos la situación se vuelve más precaria, configurando zonas que concentran a los grupos pobres y los problemas de desintegración social.

Kaztman y Retamoso (2006) hablan de un aumento de las diferencias en la calidad de los servicios y de las instituciones como uno de los efectos de la segregación que limita el acceso a fuentes de recursos; a través de los análisis por AGEB se ha visto que en las variables de agua potable, drenaje, espacios de la vivienda, servicios de salud y escolaridad, las diferencias existentes en 1990 se han ampliado para 2010, lo que lleva a decir que efectivamente las diferencias están creciendo entre las zonas de estratos bajos y las de estratos medios y altos.

Conforme han pasado los años, las diferencias físicas se mantienen, pero se han intensificado otras de carácter más socioeconómico. Hacia el final de la etapa de estabilidad económica del país, la escasez de oferta formal de suelo urbano provocó que la enorme expansión urbana superara las capacidades de los gobiernos y se ubicara sobre todo en asentamientos informales, lo que dificultaba aún más la provisión de servicios básicos, lo cual ubica a la ciudad de León en el proceso de suburbanización generalizado en América Latina, descrito por Schteingart (2010). Con los procesos de regularización implementados a partir de la década de 1990, los asentamientos han ido formalizando la situación de la tenencia de la tierra, posibilitando la introducción de servicios básicos y consolidando algunas zonas de la ciudad con enormes precariedades físicas, haciendo que estas condiciones sean cada vez menos diferenciadoras, a excepción de los estratos más bajos de la estructura socioespacial de la ciudad.

Las diferencias sociales que implican una distinción jerárquica (Castells, 1976; Rodríguez Vignoli, 2001) se han intensificado en el espacio urbano, aumentando la homogeneidad interna de los estratos bajos y las distancias con los demás estratos. Las distancias sociales presentes en una sociedad jerárquica (Bourdieu, 2002) no solo se manifiestan en todo el espacio urbano de León, sino que también se han extendido en las últimas décadas a algunas zonas de la ciudad. 
Como resultado, tenemos una tendencia de la segregación a la intensificación de sus efectos negativos hacia los estratos bajos, la segunda tendencia que la literatura plantea para este fenómeno en las últimas décadas (Kaztman, 1999; Groisman, 2010; Aguilar y Mateos, 2011).

Así pues, se confirma para la ciudad de León su inserción en los procesos recientes de la segregación (Sabatini et al., 2001; Aguilar y Mateos, 2011), ya que en las últimas dos décadas se advierte un cambio hacia una reducción de la escala de observación, así como una intensificación de los efectos negativos hacia los estratos más vulnerables, en este caso caracterizados como los estratos muy bajo y bajo de la diferenciación socioespacial, que tiene un alto componente de la situación socioeconómica de la población y sobre todo de las condiciones materiales de las viviendas y las colonias.

Asimismo, se ha podido encontrar que las características socioeconómicas van siendo cada vez menos preponderantes, para dar lugar a nuevas variables explicativas que muy probablemente estén relacionadas con los cambios sociales recientes. La capacidad explicativa y ponderación de las variables incluidas en el análisis también hace posible una lectura de las tendencias de la segregación en la ciudad. Por un lado, aunque el porcentaje de la varianza explicada por el Factor I sigue siendo relevante, este ha ido disminuyendo al pasar de 67 a $58 \%$ y finalmente a $51 \%$; esto permite decir que no obstante se puede observar una consistencia e incluso mayor intensidad de la segregación, las variables típicamente incluidas en los análisis cuantitativos están perdiendo capacidad de explicación, como lo han señalado al igual Rubalcava y Schteingart (2012), lo que da pie a la necesidad de incluir otro tipo de variables con mayor capacidad de diferenciación, aunque con la limitación de no contar con registros históricos censales. Estas nuevas variables deberían estar vinculadas con los cambios sociales más recientes, como la disponibilidad de tecnologías de información y comunicación, la expansión de mecanismos financieros más accesibles a la población, o la dimensión subjetiva de la segregación.

Los cambios en la ponderación de las variables incluidas también ofrecen una lectura en las tendencias de la segregación. Mientras que en 1990 las variables físicomateriales son más preponderantes, en 2010 lo siguen siendo pero con menor ponderación, haciendo de la segregación un fenómeno cada vez más complejo tanto para su observación como para su explicación. Si bien los procesos de consolidación física de las colonias tienen un alto componente de 
explicación para la segregación, hacia 1990 esta capacidad explicativa era más amplia, en periodos cuando la expansión urbana era más acelerada y con mayores carencias en las zonas de reciente urbanización popular.

Finalmente, estos resultados dan pie a proponer la realización de análisis diacrónicos a mayor profundidad, para identificar en un panorama más complejo la relación de estas variables socioeconómicas con otras variables que los censos no permiten realizar, así como explorar la dimensión subjetiva y, en conjunto, los efectos que la segregación está teniendo en las condiciones de vida de la población.

\section{Comentarios finales}

Si bien es deseable que un análisis diacrónico de la segregación incluya un periodo más largo que el aquí presentado (1990-2010), la disponibilidad de información en el nivel intraurbano en los censos de población se confina a este lapso, lo que impide ampliar el periodo de observación mediante la técnica de análisis factorial que aquí se utiliza. Otra limitación es que aunque las variables empleadas para definir una estratificación socioespacial que se relacionan con las condiciones físicomateriales de las viviendas y los barrios no dejan de ser relevantes, están perdiendo su capacidad de diferenciación (Rubalcava y Schteingart, 2012) y se hace necesario incluir nuevas variables que están cobrando importancia o una versión más específica de las tradicionales, pero con la restricción de que estas variables o especificidades no se encuentran en los censos anteriores, lo que dificulta el análisis diacrónico. Una vez hechas estas consideraciones, se presentan algunos comentarios finales sobre los hallazgos logrados.

Autores como Castells (2000), Sabatini (2003) y Rodríguez Vignoli (2001), entre otros, coinciden en decir que la segregación residencial es un fenómeno social en aumento. Las características particulares en las ciudades latinoamericanas se pueden apreciar en las nuevas tendencias de las últimas décadas, las cuales básicamente se presentan en dos sentidos: la reducción de la escala de la diferenciación socioespacial y la intensificación de los efectos negativos de la segregación (Sabatini et al., 2001; Aguilar y Mateos, 2011). Estas tendencias se confirman para la ciudad de León, analizadas de manera concreta con información cuantitativa para el lapso 1990-2010 a partir de tres momentos censales. 
El periodo analizado a través de las AGEB corresponde a la etapa del desarrollo urbano de la ciudad que García (2010) define como la privatización de la ciudad, caracterizada principalmente por una división social del espacio urbano. No obstante, se considera que la etapa anterior que el autor denomina de expansión y vialidades (1980-1990) aún no puede darse por concluida, ya que la mancha urbana sigue en aumento al igual que la población; si bien García (2010) menciona que después de 1990 la ciudad continúa con una expansión cada vez más dispersa, este es un rasgo fundamental de la ciudad, así como el proceso de fragmentación y segregación.

Se ha podido constatar que, en efecto, el fenómeno de la segregación residencial está intensificándose, y destacan en el proceso las siguientes tendencias: a) dispersión urbana, $b$ ) reducción de la escala de observación y c) aumento de la diferenciación entre los estratos socioespaciales. Estas tendencias se enmarcan en los procesos recientes de las ciudades latinoamericanas, por lo que se suma evidencia sobre lo que está sucediendo en las ciudades medias de México. Sin embargo, los estudios que realicen este tipo de análisis deben avanzar en la profundización de las causas, pero sobre todo en los efectos que estos procesos tienen en las condiciones de vida de la población, para con ello comprender a mayor profundidad las implicaciones que la configuración del espacio urbano tiene sobre las sociedades que las producen y transforman.

\section{Fuentes consultadas}

Aguilar, A. y Mateos, P. (2011). Diferenciación sociodemográfica del espacio urbano de la Ciudad de México. Revista EURE, 37(110), 5-30.

Alegría, T. (1994). Segregación socioespacial urbana. El ejemplo de Tijuana. Estudios Demográficos y Urbanos, 9(26), 411-428.

Aliaga, L. y Álvarez, M. J. (2010). Segregación residencial en Bogotá a través del tiempo y diferentes escalas. Estados Unidos: Lincoln Institute of Land Policy.

Arriagada, C. y Rodríguez Vignoli, J. (2003). Segregación residencial en áreas metropolitanas de América Latina: magnitud, características, evolución e implicaciones de política. Santiago de Chile: Comisión Económica para América Latina y el Caribe (Serie Población y Desarrollo, 47). 
Bourdieu, P. (2002). Condición de clase y posición de clase. Revista Colombiana de Sociología, 7(1), 119-141.

Castells, M. (1976). La cuestión urbana. México: Siglo xxi.

Castells, M. (2000). Grassrooting the space of flows. En J. Wheeler, J. Aoyama y B. Warf (eds.), Cities in the telecommunications age. The fracturing geographies (pp.18-27). Londres: Routledge.

Duhau, E. (2003). División social del espacio metropolitano y movilidad residencial. Papeles de Población, 36, 161-210.

García, M. A. (2010). Transformaciones urbanas en León, siglo xx. México: Tlacuilo Ediciones/Universidad de Guanajuato, Departamento de Arquitectura.

Germani, G. (1973). El concepto de marginalidad: significado, raíces históricas y cuestiones teóricas, con particular referencia a la marginalidad urbana. Buenos Aires: Nueva Visión.

Gómez, M. C. (2004). Métodos y técnicas de la cartografía temática III.4. México: Universidad Nacional Autónoma de México, Instituto de Geografía.

González Arellano, S. y Villeneuve, P. (2002). Desigualdad social en el espacio urbano en México. Ciudades, 53, 51-58.

Groisman, F. (2010). La persistencia de la segregación residencial socioeconómica en Argentina. Estudios Demográficos y Urbanos, 74, 429-460.

Harvey, D. (1992). Social justice, pormodernism and the city. International Journay of Urban and Regional Research, 16, 588-601.

Harvey, D. (2005). El arte de la renta: la globalización y la mercantilización de la cultura. En D. Harvey y N. Smith (coords.), Capital financiero, propiedad inmobiliaria y cultura (pp. 29-58). Barcelona: Universidad Autónoma de Barcelona.

Kaztman, R. (1999). Marginalidad e integración social en Uruguay. En J. Carpio e I. Novacovsky (comps.), De igual a igual. El desafío del Estado ante los nuevos problemas sociales (pp. 102-140). Buenos Aires: Fondo de Cultura Económica/Facultad Latinoamericana de Ciencias Sociales, Argentina.

Kaztman, R. y Retamoso, A. (noviembre, 2006). Segregación residencial en Montevideo: Desafíos para la equidad educativa. En Memorias de la Reunión de Expertos sobre Población y Pobreza en América Latina y el 
Caribe. Santiago de Chile: Comisión Económica para América Latina y el Caribe/Fondo de Población de las Naciones Unidas.

McKenzie, R. (1967). The ecological approach to the study of the human community. In R. E. Park, E. W. Burgess y R. McKenzie (eds.), The City (pp. 63-79). Chicago: University of Chicago Press.

Preteceille, E. y Queiroz Ribeiro, L. C. de (1999). Tendências da segrecação social em metrópoles globais e desiguais: Paris e Rio de Janeiro nos anos 80. Revista EURE, 25(76), 79-102.

Puebla, C. (2007). Los programas de vivienda. En S. Tamayo (coord.), Los desafíos del Bando 2. Evaluación multidimensional de las políticas habitacionales en el Distrito Federal 2000-2006 (pp. 115-164). México: Secretaría de Desarrollo Urbano y Vivienda/Instituto de Vivienda del Distrito Federal/Universidad Autónoma de la Ciudad de México/Centro de Estudios Sociales y Culturales Antonio de Montesinos.

Quijano, A. (1977). Imperialismo y marginalidad en América Latina. Lima: Mosca Azul.

Roberts, B. R. y Wilson, R. H. (eds.). (2009). Urban Segregation and Governance in the Americas. New York: Palgrave Macmillan.

Rodríguez Vignoli, J. (2001). Segregación residencial socioeconómica: ¿Qué es?, ¿Cómo se mide?, ¿Qué está pasando?, ¿Importa? Santiago de Chile: Comisión Económica para América Latina y el Caribe (Serie Población y Desarrollo, 16).

Rodríguez Vignoli, J. y Arriagada, C. (2004). Segregación residencial en la ciudad latinoamericana. Revista EURE, 29(89), 5-24.

Rubalcava, R. M. y Schteingart, M. (2000). Segregación socioespacial en el Área Metropolitana de la Ciudad de México. En G. Garza (coord.), La Ciudad de México en el fin del segundo milenio (pp. 287-296). México: Gobierno del Distrito Federal/El Colegio de México.

Rubalcava, R. M. y Schteingart, M. (2001). Segregación socioespacial en el Área Metropolitana de la Ciudad de México. En G. Garza (coord.), Atlas de la ciudad de México (pp. 108-115). México: Gobierno del Distrito Federal/El Colegio de México.

Rubalcava, R. M. y Schteingart, M. (2012). Ciudades divididas. Desigualdad y segregación social en México. México: El Colegio de México. 
Sabatini, F. (2003). La segregación social del espacio en las ciudades de América Latina. Washington, D. C.: Banco Interamericano de Desarrollo.

Sabatini, F. y Brain, I. (2008). La segregación, los guetos y la integración social urbana: mitos y claves. Revista EURE, 34(103), 5-26.

Sabatini, F., Cáceres, G. y Cerda, J. (2001). Segregación residencial en las principales ciudades chilenas: Tendencias de las tres últimas décadas y posibles cursos de acción. Revista EURE, 27(82), 21-42.

Sassen, S. (1991). The Global City. New Jersey: Princeton University Press.

Schteingart, M. (1995). Urban Research in Mexico, Colombia and Central America. An Agenda for the 90. In R. Stren (coord.), Latin America, Urban Research in the Developing World (pp. 143-221). Toronto: University of Toronto.

Schteingart, M. (2001). La división social del espacio en las ciudades. Perfiles Latinoamericanos, 19, 13-31.

Schteingart, M. (2010). División social del espacio y segregación en la ciudad de México. Continuidad y cambios en las últimas décadas. En G. Garza y M. Schteingart (coords.), Los grandes problemas de México. II. Desarrollo urbano y regional (pp. 345-387). México: El Colegio de México.

UN-Habitat (2008). State of the World's Cities 2010/2011. Bridging The Urban Divide. London: Author.

Valladares, L. y Prates, M. (1995). Urban Research in Brazil and Venezuela: Towards an Agenda for the 1990. In R. Stren (coord.), Latin America, Urban Research in the Developing World (pp. 43-142). Toronto: University of Toronto. 
